\title{
Identification of differentially expressed genes of Haloxylon ammodendron in response to salinity stress
}

\author{
J.F. HE ${ }^{1}$, Y.Y. FANG ${ }^{1}$, Z.X. LU ${ }^{3}$, L.W. WANG ${ }^{1}$, X.Q. ZHAO ${ }^{1}$, X.F. FU' ${ }^{1}$ J. ZHAO ${ }^{2 *}$, and H.K. LIU ${ }^{*}$ \\ Biotechnology Research Centre, Inner Mongolia Academy of Agriculture \& Animal Husbandry Sciences, \\ Hohhot, 010030, P.R. China ${ }^{1}$ \\ Reproductive Centre, Affiliated Hospital of Inner Mongolia Medical University, Hohhot, 010050, P.R. China ${ }^{2}$ \\ Lethbridge Research Centre, Agriculture and Agri-Food Canada, Lethbridge, Alberta T1J4B1, Canada ${ }^{3}$
}

\begin{abstract}
Haloxylon ammodendron (C.A. Mey.), an endangered desert tree with excellent drought and salinity tolerance, provides a unique genotype to characterize and understand the tolerance mechanisms. In this study, four RNA-Seq libraries were constructed and sequenced from $H$. ammodendron under salinity stress. Total 12027 differentially expressed genes (DEGs) were identified, in which 4 023, 3517,4487 genes were differentially expressed under light salinity stress $(200 \mathrm{mM} \mathrm{NaCl})$, moderate salinity stress $(400 \mathrm{mM} \mathrm{NaCl})$, and severe salinity stress $(800 \mathrm{mM} \mathrm{NaCl})$, respectively. The up-regulated DEGs included several transcription factors (e.g., $M Y B$ and bHLH), hormone-related genes (e.g., cytochrome P450), protein kinases (e.g., Atpk2-Atpk19 like), and genes involved in carbon metabolism (e.g., UDP glycosyltransferase), osmotic regulation (e.g., proline transporter), and ubiquitin proteasome system (e.g., ubiquitin-conjugating enzymes). Heat shock proteins were identified as positive regulators of salinity tolerance in H. ammodendron. The expression patterns of 13 DEGs verified by real-time quantitative PCR were identically consistent with the variations in transcript abundance identified by RNA-Seq. Our results provide new insights into molecular mechanism of H. ammodendron in response to salinity stress.
\end{abstract}

Additional key words: carbon metabolism, drought and salinity tolerance, heat shock proteins, hormone-related genes, $\mathrm{NaCl}, \mathrm{RNA}-\mathrm{Seq}$, transcription factors.

\section{Introduction}

Salinity stress is one of the main environmental factors limiting plant growth and crop productivity (Flowers 2004). The mechanisms of salt tolerance in plants can be divided into three main classes: osmotic adjustment, ion exclusion, and tissue tolerance (Roy et al. 2014). Transcript regulation in response to salinity stress and different gene expression under salinity stress have been characterized in many plants such as Arabidopsis (Jakoby et al. 2002), wheat (Niu et al. 2012), rice (Kawasaki et al. 2001, Fang et al. 2008), and maize (Andjelkovic and Thompson 2006). Recent research showed that the tissue-selective signaling and hormone crosstalk are related to the salinity stress (Wu et al. 2015, You et al. 2016). Several gene families and metabolic pathways, such as the NAC (NAM - no apical meristem, ATAF - Arabidopsis transcription activation factor, and $C U C$ - cup-shaped cotyledon) transcription factor family (Fang et al. 2008) and cation/ proton antiporters (CPA) family (Jia et al. 2018), have been characterized for their salt tolerance. In Arabidopsis, three $N A C$ genes were induced by drought, salinity, and/ or low temperature (Tran et al. 2004). Arabidopsis NAC gene $A t N A C 2$ can be induced by high salinity and the overexpression of AtNAC2 altered lateral root development and enhanced salt tolerance (He et al. 2005). The drought

Submitted 24 May 2018, last revision 25 March 2019, accepted 8 April 2019.

Abbreviations: ATAF - Arabidopsis transcription activation factor; AP2/EREBP - APETALA2/ethylene-responsive element binding proteins; bHLH - basic helix-loop-helix; bZIP - basic leucine zipper; COG - clusters of orthologous groups of proteins; CPA - cation/ proton antiporters; CUC - cup-shaped cotyledon; CYP - cytochrome protein; DEG - differentially expressed gene; GO - gene ontology; HSP - heat shock protein; MYB - myeloblastosis family of transcription factors; KEGG - Kyoto encyclopaedia of genes and genomes; LSS - light salinity stress; MSS - moderate salinity stress; NAC - NAM (no apical meristem) ATAF and CUC; qPCR - quantitative PCR; RNA-Seq - RNA-based next generation sequencing; SSS - severe salinity stress; TF - transcription factor; UPS - ubiquitin proteasome system; WRKY - containing a conserved WRKYGQK domain.

Acknowledgements: This work was supported by the Agriculture and Animal Husbandry Innovation Fund of Inner Mongolia (2017CXJJM08, 2013CXJJN15), the Inner Mongolia Natural Fund (2018MS03006, 2018LH03006), and the Inner Mongolia Education Department fund (NJZY16118). J.F. He and Y.Y. Fang contributed equally to this work.

* Corresponding authors; e-mails: zhaojie981224@163.com,229127222@qq.com 
resistance and salt tolerance in the transgenic rice are significantly improved by the over-expression of stress responsive gene $S N A C 1$ (Hu et al. 2006). The CPA family comprises $\mathrm{Na}^{+} / \mathrm{H}^{+}$exchanger $(N H X), \mathrm{K}^{+}$-efflux antiporter (KEA), and cation $/ \mathrm{H}^{+}$exchanger (CHX) (Jia et al. 2018). There are about 40 CPA genes in Arabidopsis and some of their homologues have been identified in other plant species (Jia et al. 2018). Members of the CPA gene family, such as $N H X 1$ and $N H X 2$, were reported to play a role in salt tolerance at cellular, organ, or whole-plant levels (Barragán et al. 2012). Several novel soybean CHX genes were identified to have a protective function under salinity stress (Jia et al. 2017).

Haloxylon ammodendron is a kind of xerophytic desert tree and the source of Cistanche deserticola, a precious herbal medicine. Haloxylon ammodendron has an excellent drought and salinity tolerance and plays a very important role in maintaining the ecological balance of entire desert ecosystem in which it grows (Long et al. 2014). Previous studies on $H$. ammodendron were mainly focused on its physiology or the expression of a small amount of its genes in response to salinity stress (Xie et al. 2008, Gao et al. 2010, Yang et al. 2011). However, the molecular mechanism of salinity tolerance in H. ammodendron is still unclear. In this study, the RNA-Seq data of H. ammodendrons in response to salinity stress was generated, transcriptional differences of potential salinity tolerance genes were characterized, and their putative functions were deduced in the patterns in which their transcript abundance were described under different salinity treatments. Our assembled, annotated transcriptome datasets and gene expression profiles of $H$. ammodendron will facilitate genetic and genomic studies on understanding the molecular mechanism of salinity tolerance in $H$. ammodendron as well as provide transcriptome datasets to develop new plant cultivars with novel salinity tolerance through genetic engineering.

\section{Materials and methods}

Plants and salinity treatments: Haloxylon ammodendron (C.A. Mey.) seeds were germinated in plastic pots with a sufficiently moistened Cornell Peat-Lite mix. The seedlings were grown in a growth cabinet with day/ night temperatures of $24 / 20{ }^{\circ} \mathrm{C}$, a 16 -h photoperiod, a $45 \%$ relative air humidity, and a photon flux density of $350 \mu \mathrm{mol} \mathrm{m} \mathrm{m}^{-2} \mathrm{~s}^{-1}$. A completely randomized design was used with 20 uniform plants. After three months of growth, three salinity treatments were applied: light salinity stress (LSS, $200 \mathrm{mM} \mathrm{NaCl}$ ), moderate salinity stress (MSS, $400 \mathrm{mM} \mathrm{NaCl}$ ), and severe salinity stress (SSS, $800 \mathrm{mM}$ $\mathrm{NaCl}$ ). Five plants were assigned to each treatment and the control (CK, without salinity stress). The soil salinity was kept at the predetermined levels by weighting the individual pot and rehydrating it every day. The plants stressed for three months in each treatment were sampled and sequenced.

Isolation of RNA, cDNA library construction and sequencing: To investigate the differentially expressed genes (DEGs) of $H$. ammodendron in response to salinity stress, total RNA was extracted from whole seedlings and the cDNA was reversely transcribed from mRNA of each treatment according to our previous publication (He et al. 2013). Three replicates for each treatment were applied for cDNA sequencing. The Illumina paired-end sequencing technology (Illumina Hi Seq 2000 platform) was used to sequence and analyze raw RNA-Seq data and the base calling were produced by the Illuminain software analyzer at the HUADA Gene Company (Sheng Zhen, China).

Transcriptome de novo assembly and sequence annotation: The quality of raw sequence reads for the control and three salinity treatments from the image

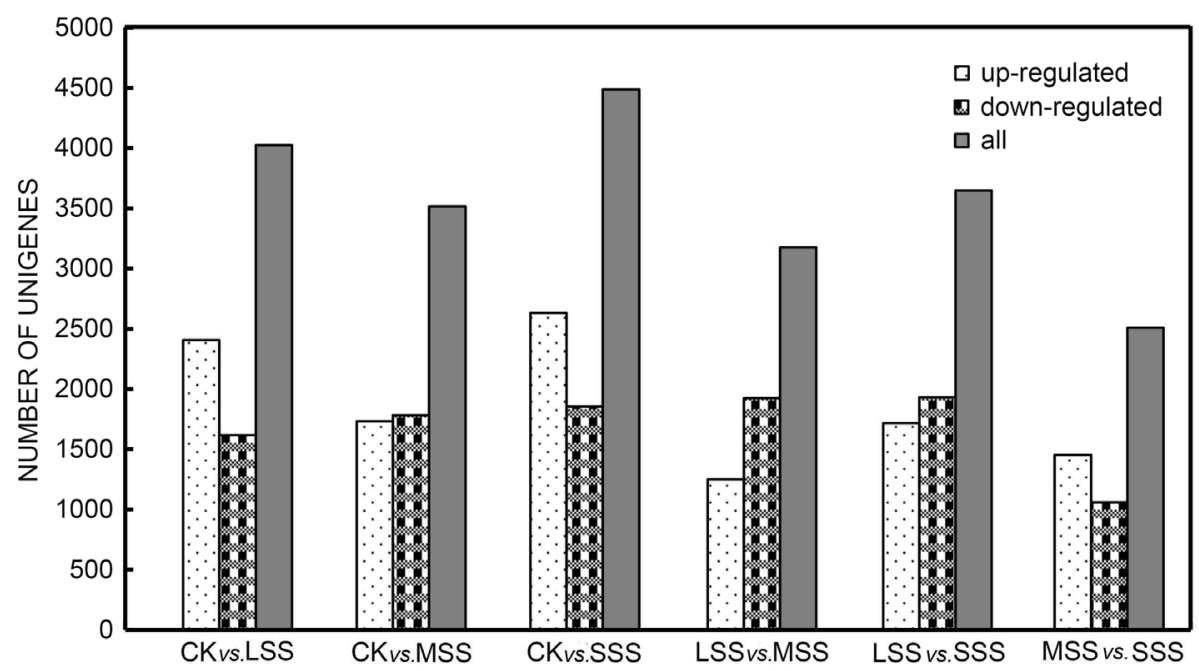

Fig. 1. Differentially expressed genes (DEGs) from six pairwise comparisons of four different RNA-Seq libraries of Haloxylon ammodendron in response to salinity stress. Up-regulated genes, down-regulated genes, and total DEGs were identified and characterized. LSS - light salinity stress (200 mM NaCl); MSS - moderate salinity stress (400 mM NaCl); SSS - severe salinity stress $(800 \mathrm{mM} \mathrm{NaCl})$. 
data output of the Illumina sequencing facility were checked by HUADA Gene Company. The ribosomal RNA contamination, low quality bases, and adapters were trimmed and removed. The de novo assembly of $H$. ammodendron transcriptomes was accomplished by following the online instructions of Trinity (http://trinityrnaseq.sourceforge.net/). Reads per kilobase per million reads was used to analyze the gene expression. To validate the assembly reliability, all unigenes were annotated through BLASTX alignment against the $\mathrm{Nr}$ or Uni Prot database and NCBI non-redundant using the BLAST 2.2.28+ program with an e-value threshold of $1 \mathrm{e}^{-5}$.

Characterization of differentially expressed genes: Read counts for each gene were calculated to identify DEGs and then were modeled as the Poisson distribution (Audic and Claverie 1997). The DEGs were identified requiring a false discovery rate of 0.001 and an absolute value of $\log 2$ (fold-change). Cluster analysis of gene expression patterns

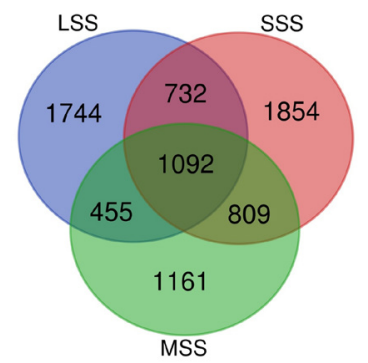

Fig. 2. The summary of Haloxylon ammodendron differentially expressed genes under three salinity treatments. LSS - light salinity stress $(200 \mathrm{mM} \mathrm{NaCl})$; MSS - moderate salinity stress (400 mM NaCl); SSS - severe salinity stress (800 mM NaCl). was performed by using the Cluster software (De Hoon et al. 2002) and Java Tree View (Saldanha 2004). To better understand the functions of these specifically regulated genes and gain more insights into these genes, BLAST analysis and gene ontology $(G O)$ analysis were performed. In the analysis of gene expression profiles, functional assignments were mapped onto $G O$ terms (Harris et al. 2004). The $G O$ enrichment analysis was constructed using WEGO (Ye et al. 2006). Significantly enriched Kyoto Encyclopedia of Genes and Genomes $(K E G G)$ pathways were identified according to the $P$ values and enrichment factors (Ye et al. 2006) using a BLAST search against the $K E G G$ database and then mapped onto $K E G G$ pathways.

Real-time quantitative PCR validation: Gene expression patterns were validated by real-time quantitative PCR (qPCR) to measure the digital transcript abundance and 13 DEGs were randomly selected and analyzed using the real-time qPCR. Primers for 13 DEGs and a reference gene were designed by the Primer 5 software based on the target genes (Table 1 Suppl.). Following the manufacturer's instructions, total RNA was treated with genomic DNA eraser to remove gDNA contamination, and then about $0.4 \mu \mathrm{g}$ of total RNA was used for cDNA synthesis by a PrimeScript $^{\mathrm{TM}} R T$ reagent kit with a gDNA eraser sample kit (TaKaRa, Tokyo, Japan). The $0.01 \mathrm{~cm}^{3}$ of quadruplet PCR reaction solutions contained $0.005 \mathrm{~cm}^{3}$ of real-time SYBR Premix Ex Taq (TaKaRa), $5 \mu \mathrm{M}$ forward primers, $5 \mu \mathrm{M}$ reverse primers, and $20 \mathrm{ng}$ of cDNA templates. All reactions were run as duplicates in 96-well plates. The quadruplet PCR reactions were performed on a Lightcycle 480 (Roche, Basel, Switzerland) using the following cycle regime: $55^{\circ} \mathrm{C}$ for $2 \mathrm{~min}, 95^{\circ} \mathrm{C}$ for $10 \mathrm{~min}$ followed by 40 cycles of $95{ }^{\circ} \mathrm{C}$ for $15 \mathrm{~s}$ and $58{ }^{\circ} \mathrm{C}$ for $1 \mathrm{~min}$. The $18 \mathrm{~S}$

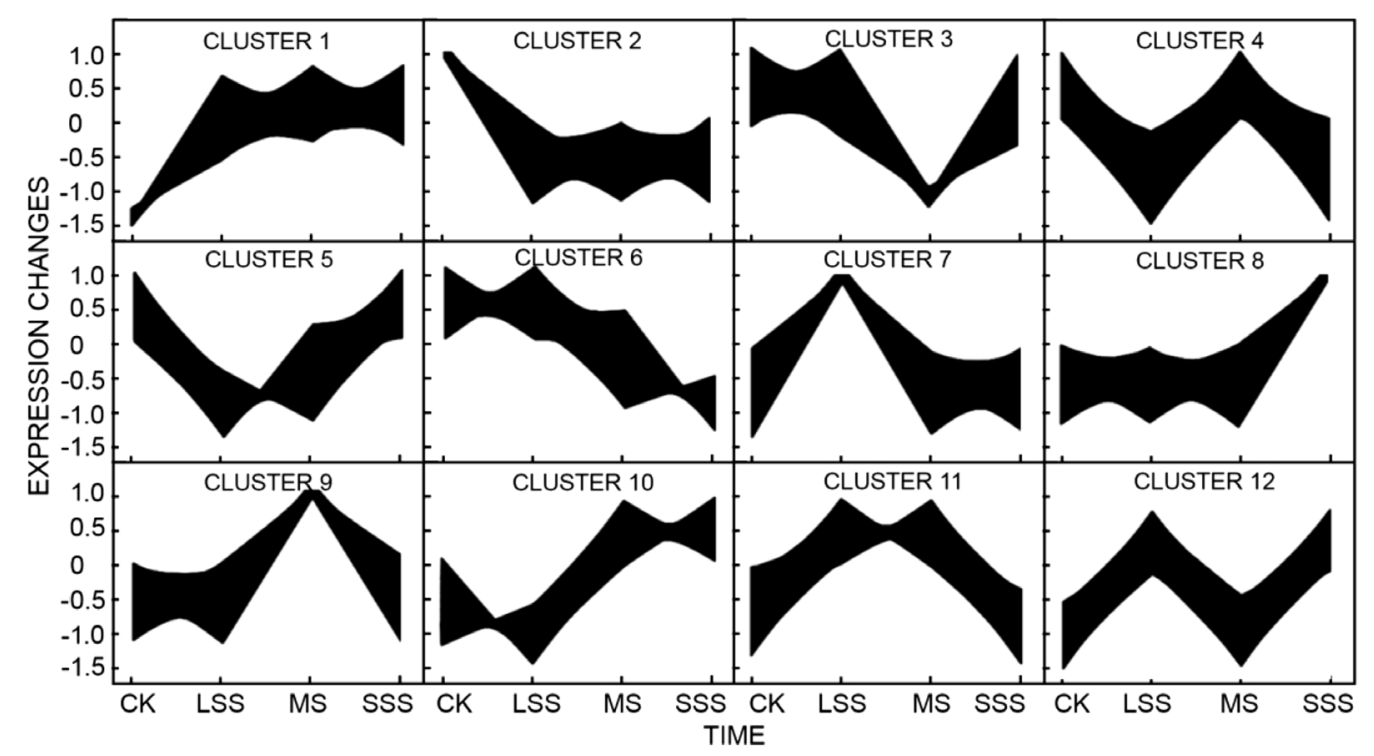

Fig. 3. Clusters and gene ontology enrichment of differently regulated genes of Haloxylon ammodendron in response to salinity stress. Genes with certain degree of regulation were grouped via hierarchical clustering. Expression values were normalized and scaled between -1.5 and 1.5 ( $y$-axis). LSS - light salinity stress (200 mM NaCl); MSS - moderate salinity stress (400 mM NaCl); $\mathrm{SSS}$ - severe salinity stress $(800 \mathrm{mM} \mathrm{NaCl})$. 


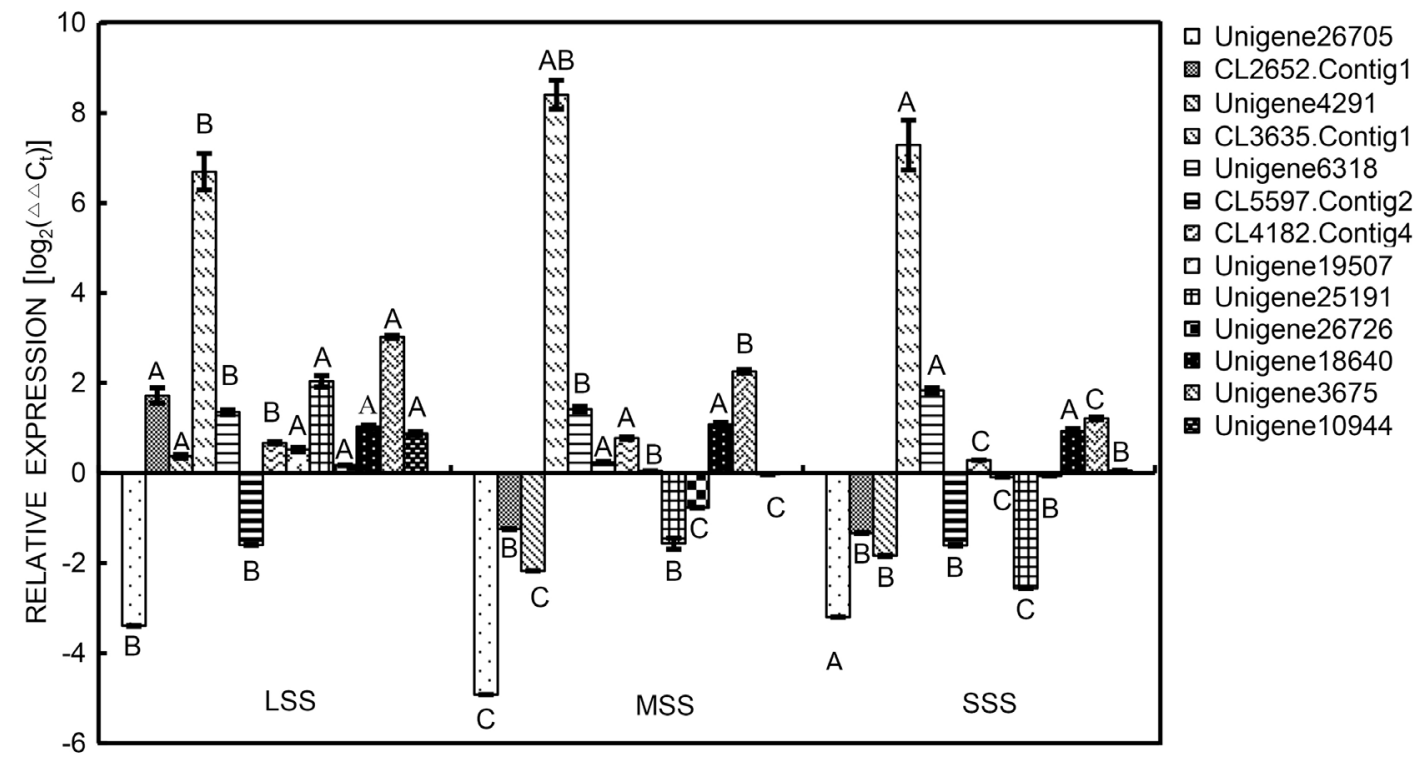

Fig. 4. Real-time PCR analysis on differently expressed genes of Haloxylon ammodendron in response to salinity stress. The $18 S$ was used to normalize the expression of all target genes. Means $\pm \mathrm{SDs}, n=3$; means with different letters are significantly different at a $1 \%$ level. LSS - light salinity stress $(200 \mathrm{mM} \mathrm{NaCl})$; MSS - moderate salinity stress $(400 \mathrm{mM} \mathrm{NaCl})$; $\mathrm{SSS}$ - severe salinity stress $(800 \mathrm{mM} \mathrm{NaCl})$.

gene was amplified in parallel as an internal reference gene. Relative expressions of the amplified products were calculated using the $2^{-\Delta \Delta C t}$ method (Kanehisa et al. 2006). Three technical replicates were used for each sample and the data are shown as means \pm standard deviations (SDs).

\section{Results and discussion}

Previous research verified that plants can enhance their salinity tolerance through modulation of gene transcription (Li et al. 2016). In this study, DEGs in H. ammodendron were analyzed under different salinity treatments and identified by pairwise comparisons of four RNA-Seq libraries, i.e., CK vs. LSS, CK vs. MSS, CK vs. SSS, LSS $v s$. MSS, LSS vs. SSS, and MSS vs. SSS (Fig. 1). Our results indicated that the largest number of DEGs (4 487) were identified from the comparison of CK vs. In SSS, 2631 genes were up-regulated and 1856 genes were down-regulated. The second largest number of DEGs (4 023) was identified from the comparison of CK vs. LSS, in which 2406 genes were up-regulated and 1617 downregulated. The third largest number of DEGs (3 647) was detected from the comparison of LSS vs. SSS, followed by CK vs. MSS (3 517), LSS vs. MSS (3 147), and MSS vs. SSS (2 510). We found that 455 DEGs were overlapped in LSS and MSS, 732 DEGs were overlapped in LSS and SSS, and 809 DEGs were overlapped in MSS and SSS (Fig. 2). Total 1092 DEGs were identified to be differently regulated under all 3 salinity treatments indicating that these stress-responsive genes may be potentially involved in biological process and/or metabolic pathways for salinity tolerance in $H$. ammodendron.
To investigate the co-expressed genes in response to salinity stress, we applied the statistical clustering analysis to all genes that were differentially expressed under three salinity treatments compared to the control. Total 12 clusters of regulated genes were detected, comprising 61599 genes that showed some degree in their different expressions (Fig. 3). The largest group (Cluster 8) included 8544 genes $(13.87 \%)$ that had no significant changes in response to LSS $(200 \mathrm{mM} \mathrm{NaCl})$ and MSS (400 mM $\mathrm{NaCl})$, but a significant increase in response to SSS $(800$ $\mathrm{mM} \mathrm{NaCl}$ ). The second largest group (Cluster 7) contained 7495 genes $(12.16 \%)$ that increased in response to LSS but continually decreased in response to MSS and SSS. The third largest group (Cluster 9) contained 6582 genes $(10.68 \%)$ and their expressions did not change notably in response to LSS and SSS except for a significant increase in response to MSS. The fourth largest group (Cluster 2) contained 6346 genes $(10.03 \%)$, with a significant decrease in response to LSS and then they were stable in response to MSS and SSS. The fifth largest group (Cluster 10) contained 5929 genes $(9.62 \%)$ with a slight decrease in response to LSS, but a significant increase in response to MSS stress and then were stable in response to SSS. The smallest group (Cluster 6) contained 3586 genes (5.82\%), and their expressions did not notably change in LSS, but continually decreased in response to MSS and SSS.

To validate the RNA-Seq-based gene expressions, realtime qPCR was performed on 13 randomly selected genes with different expressions in different salinity treatments. The qPCR results of these tested DEGs showed a similar trend that was observed in RNA-Seq data although the magnitudes of these gene expressions were different (Fig. 4). By comparing to the control, we found that the gene 
CL3635.Contig1 was the most significantly up-regulated in all three salinity treatments, following by Unigene 3675 , Unigene6318, and Unigene18640, whereas Unigene26705 was significantly down-regulated in all salinity treatments used.

Transcription factors (TFs) are important upstream regulatory proteins and play significant roles in plant responses to abiotic and biotic stresses. The role of various TFs, such as MYB (the myeloblastosis family of transcription), NAC, bHLH (basic helix-loop-helix), AP2/ EREB (APETALA2/ethylene-responsive element binding proteins), WRKY (containing a conserved WRKYGQK domain), and bZIP (basic leucine zipper), in regulating salinity and drought responses has been previously reviewed (Jiang et al. 2017). The over-expression of these transcription factors can enhance the drought or salinity tolerance of transgenic plants (Seki et al. 2002, Jeong et al. 2010, Takasaki et al. 2010, Hao et al. 2011). In this study, we found that many TFs, such as $3 A P 2-E R E B$ TFs (Unigene13909, Unigene9944, CL4042.Contig2), bHLHs (CL213.Contig1), bHLH74 (CL350.Contig2), bZIP (Unigene24130), C3H (CL2305.Contig2), 3 MYBs (CL6074.Contig2, Unigene14210, Unigene10242), and $N A C$ (Unigene11417), were significantly up-regulated under all salinity treatments (Table 2 Suppl.). Ethyleneresponsive transcription factors ERTF003 (Unigene17144) and ERTF034-like (CL4042.Contig2) were up-regulated in different salinity treatments, however, ERTF012 (Unigene3675) was down-regulated under salinity stress. Our results indicate that some ethylene-responsive TFs may function as positive or negative regulators of salinity tolerance in $H$. ammodendron.

Previous studies indicated that a number of hormonerelated genes, such as cytochrome P450 in ABA biosynthesis pathway, are significantly up-regulated in response to salinity stress (Mao et al. 2013, Zhang et al. 2014, Wang et al. 2017). There are 244 cytochrome 450 (CYP450) genes in Arabidopsis genome and the transfer of Arabidopsis AtCYP78A7 into rice can increase the drought tolerance of transgenic rice (Nam et al. 2013). Other studies indicated that the disruption of CYP707A3 in Arabidopsis can increase the drought tolerance, but the over-expression of this gene can reduce drought tolerance (Bak et al. 2011) suggesting that CYPS in different plants can be positive or negative regulators of drought tolerance. In this study, we found that salinity stress induced the transcript increases of CYP genes, such as CYP450 (Unigene20778), CYP45086B1-like (Unigene15178), and CYP450-82C4-like (Unigene20780), indicating that the CYPS may be positive regulators of salinity tolerance in $H$. ammodendron (Table 2 Suppl.).

Responses of carbon or starch metabolism to drought stress have been found in many plants such as alfalfa (Naya et al. 2007) and maize (Kakumanu et al. 2012). In $H$. ammodendron, one sugar transport protein (Unigene 7883) and four sucrose UDP glucosyltransferase proteins, i.e., UDP-glycosyl transferase 85A2-like (CL4049.Contig2), UDP-glycosyltransferase 85A3like (Unigene23953), UDP-glycosyltransferase 85A2like (CL1406.Contig1), and UDP-glycosyl transferase
(Unigene35723), were significantly up-regulated under all salinity treatments (Table 2 Suppl.). These expression data suggest that sugar or carbon metabolism as well as various sugar-related signaling pathways are influenced by salinity stress in $H$. ammodendron. Salinity tolerance of most plants is consistent with the transport of substances involved in osmotic adjustment (such as proline and other amino acids) (Cockburn et al. 1996, Chang et al. 2014). In yeast, amino acid permeases modulate the efficient transport of proline, alanine, and valine, but the expression of most amino acid permease gene family members is repressed under high salinity (Rentsch et al. 1996). Our results show that the transcription of amino acid permease 2-like (CL2149.Contig1) was induced in NaCl-treated $H$. ammodendron. We found the continuous increase in the expressions of proline iminopeptidase (CL4680.Contig2), solute carrier family 25 (CL5895.Contig2), solute carrier family 13 (CL5828.Contig1), solute carrier family 40 (CL2892.Contig1), solute carrier family 15 (CL602. Contig1), and solute carrier family 2 (CL2997 contig3) in response to salinity stress (Table 2 Suppl.). Therefore, $H$. ammodendron may have the distinct ability to synthesize and transport various osmotic protectants, such as proline, osmotin, and amino acids, to maintain cell pressure potential under osmotic stress, which has not been reported in the Arabidopsis or other eukaryotes.

Heat shock proteins (HSPs) can regulate the activity of $\mathrm{Na}^{+}$or $\mathrm{K}^{+}$-ATP enzymes. Many HSP genes are generally not expressed, but can be expressed if they are stimulated by external stimuli. Therefore, HSPS are important substances for defensing the external stimulus. In this study, a heat shock cognate $70 \mathrm{kDa}$ protein isoform1 (CL2574. Contig13), a heat shock $70 \mathrm{kDa}$ protein (CL801.Contig2), a small heat shock $17.4 \mathrm{kDa}$ protein (Unigene11667), a small heat shock protein (Unigene8639), a small heat shock protein.18.1 (Unigene22310) and a heat shock protein 83-like (CL4411.Contig1) were significantly up-regulated under different salinity treatments (Table 2 Suppl.) indicating that $H S P$ s may be a positive regulators of salinity tolerance in $H$. ammodendron.

Phosphorylation of protein kinases can activate many target proteins in response to osmotic stress in higher plants (Umezawa et al. 2004). In this study, a serinethreonine kinase (Unigene22252), a CBL-interacting serine/threonine-protein kinase 6-like protein (CL4751. Contig1), and L-type lectin-domain-containing receptor kinases were differentially up-regulated under different salinity treatments, which is consistent with the upregulated expression pattern of a Glycine soja G-type lectin S-receptor-like serine/threonine-protein kinase (GsSRK) that is significantly increased under $\mathrm{NaHCO}_{3}$ treatment (Ge et al. 2010) and under $\mathrm{NaCl}$ and polyethylene glycol (PEG) treatments (Sun et al. 2013). Our results indicate that the serine/threonine-protein kinase Atpk2-Atpk19 like (CL1544.Contig2) was significantly up-regulated in response to salinity stress similarly to the previous study that the AtLPK1 expression in Arabidopsis is strongly induced by stress hormones and salinity treatments, and the AtLPK1 over-expression in Arabidopsis can enhance salinity tolerance (Huang et al. 2013). However, the serine/ 
threonine-protein kinase sx32 (Unigene18472) was downregulated under different salinity treatments in this study. Therefore, serine-threonine kinases can have a positive or negative effect to the abiotic stress. In H. ammodendron, a large number of protein kinases may play an essential role in sensing external salinity signals and regulating gene expression at the cellular level, however, the genes encoding protein kinases in response to stresses (e.g., salinity or drought) are different from other plants.

Ubiquitin proteasome system (UPS), an important regulator of a series of life processes within a cell, is closely related to the occurrence and development of diseases (Powell et al. 2012). The energy produced by cellular metabolism can stimulate cells to replicate themselves and complete the self-metabolism and repair functions. In this study, ubiquitin extension protein (CL7928.Contig2) ubiquitin-conjugating enzyme E2 (CL4959.Contig2), ubiquitin-conjugating enzyme (CL5816.Contig1), E3 ubiquitin-protein ligase AIP2-like (Unigene10144,) and E3 ubiquitin-protein ligase UPL1-like (CL448.Contig1) were significantly up-regulated under different salinity treatments (Table 2 Suppl.). Our results indicate the ubiquitin-conjugating enzyme can promote the salinity stress at various degrees. As few studies related to ubiquitin-conjugating enzymes in response to the abiotic stresses have been reported, it would be valuable to further characterize and understand the structures and functions of ubiquitin genes newly identified in this study.

Resistance proteins contain an appropriate avirulence protein, and it guards the plant against pathogens and a direct or indirect interaction with the avirulence protein, consequently, triggering a defense system which restricts the pathogen growth. In this study, most disease resistance protein genes, such as disease resistance RPP13-like protein 1-like (Unigene2395), disease resistance protein $R G A 3$-like (Unigene5608), disease resistance protein $R G A 4$-like (unigene25823), disease resistance protein $R G A 1$ (Unigene2076), disease resistance protein $R P M 1$ (CL8031.Contig1), and disease resistance protein RPS2 (Unigene527), were significantly down-regulated under different salinity treatments indicating that the disease resistance of $H$. ammodendron was weakened by the salinity stress.

\section{Conclusions}

In this study, the transcriptome data for $H$. ammodendron obtained by RNA-Seq was used to identify and annotate a large number of DEGs regulated under salinity stress, which provides an excellent abundant dataset for further genetic and genomic research. Genes responsible for salinity stress and their expression profiles under different salinity treatments were further analyzed. The up-regulated DEGs that are related to salinity tolerance include some previously reported genes (e.g., $M Y B, b H L H, C 3 H$, and $A P 2 E R E B P$ ) and some newly identified genes (e.g., those encoding cytochrome P450, ubiquitin-conjugating enzymes, and resistance proteins). Furthermore, a certain amount of target candidate genes would serve as prospective functional studies on the salinity stress process. We found the significant changes of carbon metabolic pathways, hormone-related genes, TFs, protein kinases, and UPS in $H$. ammodendron under salinity stress. These changes mainly worked as the upstream of salinity responses and then led to hormone signalling changes, which further triggered TF expression changes and salinity tolerance in H. ammodendron. Our results provide new insights into the molecular mechanism of $H$. ammodendron in response to salinity stress and can be used as a valuable reference or resource to further characterize candidate genes potential for the development of new plant genotypes with novel salinity tolerance through genetic engineering.

\section{References}

Andjelkovic, V., Thompson, R.: Changes in gene expression in maize kernel in response to water and salt stress. - Plant Cell Rep. 25: 71-79, 2006.

Audic, S., Claverie, J.M.: The significance of digital gene expression profiles. - Genome Res. 7: 986-995, 1997.

Bak, S., Beisson, F., Bishop, G., Hamberger, B., Höfer, R., Paquette, S., Werck-Reichhart, D. (ed.): The Arabidopsis Book - Cytochromes P450. - The American Society of Plant Biologists, Washington 2011.

Barragán, V., Leidi, E.O., Andrés, Z., Rubio, L., De Luca, A., Fernández, J.A., Cubero, B., Pardo, J.M.: Ion exchangers NHX1 and NHX2 mediate active potassium uptake into vacuoles to regulate cell turgor and stomatal function in Arabidopsis. - Plant Cell 24: 1127-1142, 2012.

Chang, B., Yang, L., Cong, W., Zu, Y., Tang, Z.: The improved resistance to high salinity induced by trehalose is associated with ionic regulation and osmotic adjustment in Catharanthus roseus. - Plant Physiol. Biochem. 77: 140-148, 2014.

Cockburn, W., Whitelam, G.C., Broad, A., Smith, J.: The participation of phytochrome in the signal transduction pathway of salt stress responses in Mesembryanthemum crystallinum L. - J. exp. Bot. 47: 647-53, 1996.

De Hoon, M.J.L., Imoto, S., Satoru, M.: Open source clustering software. - Bioinformatics 20: 250-251, 2002.

Fang, Y.J., You, J., Xie, K., Xie, W.B., Xiong, L.Z.: Systematic sequence analysis and identification of tissue-specific or stress-responsive genes of NAC transcription factor family in rice. - Mol. gen. Genomics 280: 547-563, 2008.

Flowers, T. J: Improving crop salinity tolerance. - J. exp. Bot. 55: 307-319, 2004.

Gao, S., Su, P., Yan, Q., Ding, S.: Canopy and leaf gas exchange of Haloxylon ammodendron under different soil moisture regimes. - Sci. China Life Sci. 6: 718-728, 2010.

Ge, Y., Li, Y., Zhu, Y.M., Bai, X., Lv, D.K., Guo, D., Ji, W., Cai, H.: Global transcriptome profiling of wild soybean (Glycine soja) roots under $\mathrm{NaHCO}_{3}$ treatment. - BMC Plant Biol. 6: 718-728, 2010.

Hao, Y.J., Wei, W., Song, Q.X., Chen, H.W., Zhang, Y.Q., Wang, F., Zou, H.F., Lei, G., Tian, A.G., Zhang, W.K., Ma, B., Zhang, J.S., Chen, S.Y.: Soybean NAC transcription factors promote abiotic stress tolerance and lateral root formation in transgenic plants. - Plant J. 68: 302-313, 2011.

Harris, M.A., Clark, J., Ireland, A., Lomax, J., Ashburner, M., Foulger, R.: The Gene ontology (GO) data-base and informatics resource. - Nucl. Acids Res. 32: 258-261, 2004.

He, J.F., Goyal, R.K., Laroche, A., Zhao, M.G., Lu, Z.X.: Effects of salinity stress on starch morphology, composition and 
physicochemical properties during grain development in triticale. - Can. J. Plant Sci. 93: 765-777, 2013.

He, X.J., Mu, R.L., Cao, W.H., Zhang, Z.G., Zhang, J.S., Chen, S. Y.: AtNAC2, a transcription factor downstream of ethylene and auxin signaling pathways, is involved in salt stress response and lateral root development. - Plant J. 44: 903-916, 2005.

Hu, H., Dai, M., Yao, J., Xiao, B., Li, X., Zhang, Q., Xiong, L.: Overexpressing a NAM, ATAF, and CUC (NAC) transcription factor enhances drought resistance and salt tolerance in rice. Proc. nat. Acad. Sci. USA 103: 12987-12992, 2006.

Huang, P., Ju, H.W., Min, J.H., Zhang, X., Kim, S.H., Yang, K.Y.: Overexpression of L-type lectin-like protein kinase 1 confers pathogen resistance and regulates salinity response in Arabidopsis thaliana. - Plant Sci. 203: 98-106, 2013.

Jakoby, M., Weisshaar, B., Dröge-Laser, W., Vicente-Carbajosa, J., Tiedemann, J., Kroj, T., Parcy, F.: bZIP transcription factors in Arabidopsis. -Trends Plant Sci. 3: 106-111, 2002.

Jeong, J.S., Kim, Y.S., Baek, K.H., Jung, H., Ha, S.H., Choi, Y.D., Kim, J.K.: Root-specific expression of OsNAC10 improves drought tolerance and grain yield in rice under field drought conditions. - Plant Physiol. 153: 185-197, 2010.

Jia, B.W., Sun, M.Z., Duanmu, H.Z., Ding, X.D., Zhu. Y.M., Sun, X.L.: GsCHX19.3, a member of cation/H+ exchanger superfamily from wild soybean contributes to high salinity and carbonate alkaline tolerance. - Sci. Rep. 7: 9423, 2017.

Jia, Q., Zheng, C., Sun, S., Amjad, H., Liang, K., Lin, W.: The role of plant cation/proton antiporter gene family in salt tolerance. - Biol. Plant. 62: 617-629, 2018.

Jiang, J.J., Ma, S.H., Ye, N.H., Jiang, M., Cao, J.S., Zhang, J.H.: WRKY transcription factors in plant responses to stresses. - J. integr. Plant Biol. 59: 86-101, 2017.

Kakumanu, A., Ambavaram, M.M.R., Klumas, C., Krishnan, A., Batlang, U., Myers, E.: Effects of drought on gene expression in maize reproductive and leaf meristem tissue revealed by RNA-Seq. - Plant Physiol. 160: 846-867, 2012.

Kanehisa, M., Goto, S., Hattori, M., Aoki-Kinoshita, K.F., Itoh, M., Kawashima, S.: From genomics to chemical genomics: new developments in KEGG. - Nucl. Acids Res. 34: 354-357, 2006.

Kawasaki, S., Borchert, C., Deyholos, M., Wang, H., Brazille, S., Kawai, K., Galbraith, D., Bohnert, H.J.: Gene expression profiles during the initial phase of salt stress in rice. - Plant Cell 13: 889-905, 2001.

Li, S.F., Fan, L.C.M., Li,Y., Zhang, J.H., Sun, J.H., Chen, Y.H., Tian, C.Y., Su, X.H., Lu, M.Z., Liang, C.Z., Hu, Z.M.: Effects of drought and salt-stresses on gene expression in Caragana korshinskii seedlings revealed by RNA-Seq. - BMC Genomics 17: 200-209, 2016.

Long, Y., Zhang J.W., Tian, X.J., Wu, S.S., Zhang. Q., Zhang, J.P., Dang, Z.H., Pei, X.W.: De novo assembly of the desert tree Haloxylon ammodendron (C.A. Mey) based on RNA-Seq data provides insight into drought response, gene discovery and marker identification. - BMC Genomics 15: 1471-2164, 2014.

Mao, G., Seebeck, T., Schrenker, D., Yu, O.: CYP709B3, a cytochrome P450 monooxygenase gene involved in salt tolerance in Arabidopsis thaliana. - BMC Plant Biol. 13: 169$181,2013$.

Nam, K.H., Nam, K.J., An, J.H., Jeong, S.C., Park, K.W.: Comparative analysis of key nutrient composition between drought-tolerant transgenic rice and its non-transgenic counterpart. - Food Sci. Biotechnol. 22: 1-7, 2013.

Naya, L., Ladrera, R., Ramos, J., González, E.M., Arrese-Igor, C., Minchin, F.R.: The response of carbon metabolism and antioxidant defenses of alfalfa nodules to drought stress and to the subsequent recovery of plants. - Plant Physiol. 144: 1104-1114, 2007.

Niu, C.F., Wei, W., Zhou, Q.Y., Tian, A.G., Hao, Y.J., Zhang, W.K.: Wheat WRKY genes TaWRKY2 and TaWRKY19 regulate abiotic stress tolerance in transgenic Arabidopsis plants. - Plant Cell Environ. 35: 56-70, 2012.

Powell, S. R., Herrmann, J., Lerman, A., Patterson, C., Wang, X. J.: The ubiquitin- proteasome system and cardiovascular diseases. - Prog. Mol. Biol. Transl. Sci. 109: 295-346, 2012.

Rentsch, D., Hirner, B., Schmelzer, E., Frommer, W.B.: Salt stress-induced proline transporter and salt stress-repressed broad specificity amino acid permeases identified by suppression of a yeast amino acid permease targeting mutant. - Plant Cell. 8: 1437-1446, 1996.

Roy, S.J., Negrão, S., Tester, M.: Salt resistant crop plants. - Curr. Opin. Biotechnol. 26: 115-124, 2014.

Saldanha, A.J.: Java Tree View - extensible visualization of microarray data. - Bioinformatics 20: 3246-3248, 2004.

Seki, M., Narusaka, M., Ishida, J., Nanjo, T., Fujita, M., Oono, Y.: Monitoring the expression profiles of 7000 Arabidopsis genes under drought, cold and high salinity stresses using a full-length cDNA microarray. - Plant J. 31: 279-292, 2002.

Sun, X.L., Yua, Q.Y., Tang, L.L., Ji, W., Bai, X., Cai, H.: GsSRK, a G-type lectin S-receptor-like serine/threonine protein kinase, is a positive regulator of plant tolerance to salt stress. - J. Plant Physiol. 170: 505-515, 2013.

Takasaki, H., Maruyama, K., Kidokoro, S., Ito, Y., Fujita, Y., Shinozaki, K.: The abiotic stress-responsive NAC-type transcription factor OsNAC5 regulates stress-inducible genes and stress tolerance in rice. - Mol. gen. Genomics 284: 173183. 2010.

Tran, L.S., Nakashima, K., Sakuma, Y., Simpson, S.D., Fujita, Y., Maruyama, K., Fujita, M., Seki, M., Shinozaki, K., Yamaguchi-Shinozaki, K.: Isolation and functional analysis of Arabidopsis stress inducible NAC transcription factors that bind to a drought responsive cis-element in the early responsive to dehydration stress 1 promoter. - Plant Cell 16: 2481-2498, 2004.

Umezawa, T., Yoshida, R., Maruyama, K., Yamaguchi-Shinozaki, K., Shinozaki, K.: SRK2C, a SNF1-related protein kinase 2, improves drought tolerance by controlling stress-responsive gene expression in Arabidopsis thaliana. - Proc. natl. Acad. Sci. USA 101: 17306-17311, 2004.

Wang. P., Ma, L., Y.L., Wang, S.: Transcriptome analysis reveals sunflower cytochrome P450 CYP93A1 responses to high salinity treatment at the seedling stage. - Genes Genomics 6: 581-591, 2017.

Wu, H., Shabala, L., Liu, X., Azzarello, E., Zhou, M., Pandolfi, C., Bose, J., Chen, Z.H., Mancuso, S., Shabala S.: Linking salinity stress tolerance with tissue-specific $\mathrm{Na}^{+}$sequestration in wheat roots. - Front. Plant Sci. 6: 71, 2015.

Xie, T.T., Zhang, X.M., Liang, S.M., Shan, L.S., Yang, X.L., Hua, Y.H.: Effects of different irrigations on the water physiological characteristics of Haloxylon ammodendron in Taklimakan desert hinterland. - Ying Yong Sheng Tai Xue Bao 19: 711-716, 2008. [In Chin.]

Yang, Y.F, Zhou, H.F, Xu, L.G.: Dynamic variations of soil moisture in Haloxylon ammodendron root zone in Gurbantunggut desert. - Ying Yong Sheng Tai Xue Bao 22: 1711-1716, 2011. [In Chin.]

Ye, J., Fang, L., Zheng, H.K., Zhang, Y., Chen, J., Zhang, Z.J.: WEGO: a web tool for plotting GO annotations. - Nucl. Acids Res. 34: 293-297, 2006.

You, Q., Zhang, L., Yi, X., Zhang, K., Yao, D., Zhang, X., Wang, Q., Zhao, X., Ling, Y., Xu, W., Li, F., Su, Z.: Co-expression network analyses identify functional modules associated with 
development and stress response in Gossypium arboretum. Sci. Rep. 6: 1-15, 2016.

Zhang, J., Feng, J.J., Lu, J., Yang, Y.Z., Zhang, X., Wan, D.S.,
Liu, J.Q.: Transcriptome differences between two sister desert poplar species under salt stress. - BMC Genomics 15: 337 350, 2014. 